\title{
Theory and Experiments of a Rolling-ball Mechanism for Horizontal
}

\section{Vibration Isolation}

\author{
Gaofeng Guan ${ }^{\mathrm{a}}{ }^{*}$ Qiang Yu ${ }^{\mathrm{b}}$ Fei Shen ${ }^{\mathrm{c}}$
}

\author{
School of Mechanical and Electronic Engineering, University of Electronic Science and Technology \\ of China, Chengdu, SiChuan, China
}

agyjj2008@aliyun.com, byuqiang199104@163.com, cshenflying@hotmail.com

Keywords: rolling-ball; vibration isolation; dynamic equation; natural frequency

\begin{abstract}
It's significant to isolate micro-vibration interference from ground to precision instruments, so lots of scholars have paid attention to study vibration isolation mechanisms and got a fruitful outcome. The most important characteristics of a passive vertical vibration isolator are natural frequency and payload capacity while horizontal vibration isolator only concerns the former. So the performance of a passive vibration isolator in low input frequencies is considerably improved by reducing its natural frequency. This paper studied a rolling-ball mechanism that the ball rolls relative to two ball sockets with unequal radii. When the motion was assumed as slight movement, according to relational geometric relationship, the Lagrange dynamics equation of rolling angle $\beta$ as generalized coordinate was established. The horizontal natural frequency has been also obtained. Then the simulation analysis of influences of various parameters on natural frequency was studied. Finally, low natural frequency has been realized in the experiments which confirmed its validity.
\end{abstract}

\section{Introduction}

In order to achieve a better performance during the use in the field of precision instruments, high-end biological and chemical experiments or optical apparatus, the devices should be installed in foundation platform that can isolate micro-vibration disturbance [1].Passive isolators is most widely used in vibration elimination as they are low cost, easy to implement and so on. The vibration isolation performance of a passive vibration isolator in low input frequencies is considerably improved by reducing its natural frequency, and lower frequency means better performance $[2,3]$.

Non-independent isolators are often composed of several regularly arranged vibration isolation mechanisms with the same basis, where rolling types of horizontal vibration isolation mechanisms have attracted the attention of many scholars. Lin [4] presented a free rolling mechanism to isolate seismic damage to buildings of which a sphere was placed between two smooth surfaces, relying on rolling friction to consume energy to the superstructure, but it could not restore to the initial position and limit horizontal displacement. Zhou [5] proposed an improved way that placed the ball between two ball sockets, and earthquake simulation tests confirmed that it had a better seismic isolation performance. Jiangid [6,7] proposed two improved methods about free rolling mechanism, one is to replace the ball with ellipsoid, and the other is to use parallel with cantilever beam or spring. YasudaA [8] also proposed another free rolling mechanism, he replaced the upper and lower areas of the ball with a part of sphere of unequal radii, respectively. Butterworth [9] carried out a detailed theoretical modeling and parameters analysis, and equivalent linearization of stiffness of natural frequency of 
YasudaA's improved mechanism, finally, simulation analysis confirmed its effectiveness. Harvey [10, 11] has investigated numerically the chaotic response of a rolling mechanism with curved slots of varied radius and rich chaotic behavior has exhibited in the case where the response includes impacts.

Based on the General, a rolling ball mechanism with an upper and lower ball sockets with nonequal radii is studied in this paper. The natural frequency has been obtained by theoretical analysis and the simulation shows how each parameter to influence on the natural frequency. Then the mechanism was applied to precision vibration isolation to suppress the slight vibrations of the ground. The experimental results verifies that the institution could achieve a lower frequency, which confirmed that the vibration isolation system is efficient.

\section{Theoretical analysis}

\subsection{The basic configuration}

The basic configuration of the vibration isolation mechanism is showed in Fig. 1(a). It is composed of an upper plate, a ball and a lower plate, and the upper plate and the lower plate both have a ball socket called upper ball socket and lower ball socket.

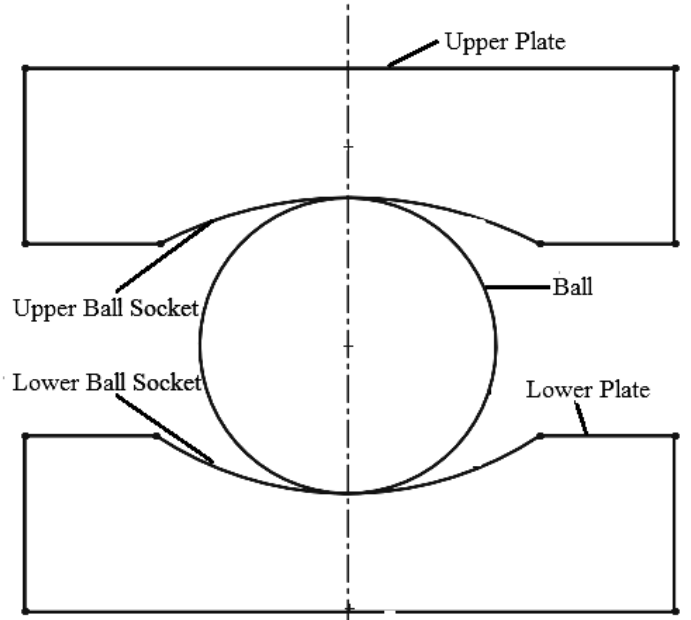

(a)

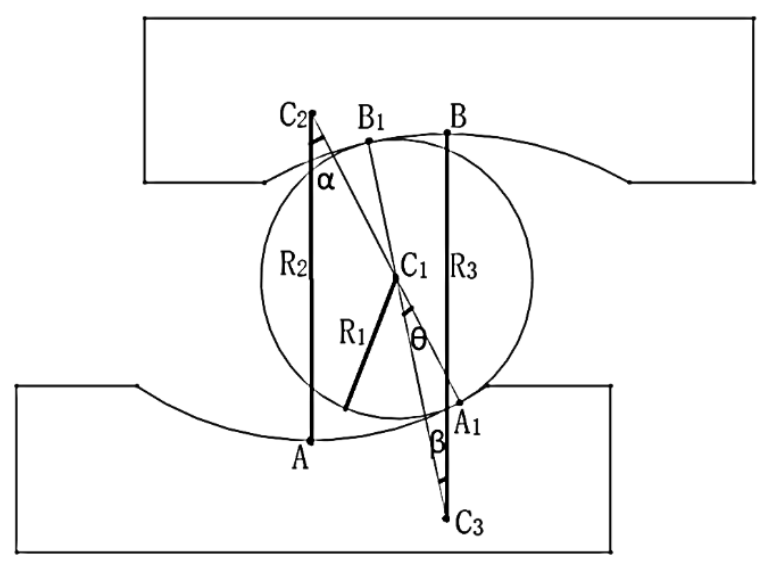

(b)

Fig.1 (a) Diagram of the mechanism in balanced state; (b) Diagram of the mechanism in motion state

\section{Table 1}

The parameters of the mechanism in Fig.1 and Fig. 2.

\begin{tabular}{ll}
\hline Symbol & Description \\
\hline $\mathrm{C}_{1}$ & The center of the ball \\
$\mathrm{C}_{2}$ & The center of the lower ball socket \\
$\mathrm{C}_{3}$ & The center of the upper ball socket \\
$\mathrm{R}_{1}$ & The radius of the ball \\
$\mathrm{R}_{2}$ & The radius of the lower ball socket \\
$\mathrm{R}_{3}$ & The radius of the upper ball socket \\
$\mathrm{A}$ & The contact point of the ball and the lower ball socket in \\
& balanced state \\
$\mathrm{B}_{1}$ & The contact point of the ball and the upper ball socket in \\
$\mathrm{A}_{1}$ & The contact point of the ball and the lower ball socket in \\
& motion state
\end{tabular}




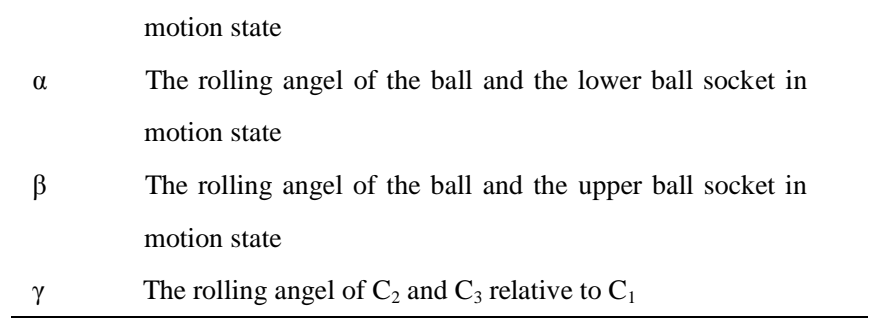

\subsection{Geometric constraints and relationships}

To be general, we assume that:

(1) The radii of the ball sockets in the upper and lower plates are unequal, such as $R_{3}>R_{2}$;

(2) The upper and lower ball sockets and the ball are rigid, without distortion;

(3) The motion type of the ball relative to the ball sockets in the lower and upper plates is rolling without sliding.

As shown in Fig. 1(a), when the mechanism is in balance state, the centers of the ball sockets in the upper plate and lower plate and the center of the ball are in the same vertical line.

As shown in Fig. 1(b), whenever the mechanism is in the motion state and the ball rolls relative to the lower and the upper ball sockets, the center of the upper ball socket $\mathrm{C}_{3}$, the center of ball $\mathrm{C}_{1}$ and the contact point of the ball and the upper ball socket $\mathrm{B}_{1}$ are in the same line, so as the center of the lower ball socket $\mathrm{C}_{2}$, the center of the ball $\mathrm{C}_{1}$ and the contact point of the ball and the lower ball socket $\mathrm{C}_{2}$. $\mathrm{A}$ is the contact point of the ball and the lower ball socket and $\mathrm{B}$ is the contact point of the ball and the upper ball socket in balanced state, and $\mathrm{A}_{1}$ is the contact point of the ball and the lower ball socket and $\mathrm{B}_{1}$ is the contact point of the ball and the upper ball socket in motion state.

The arc concave $\mathrm{AA}_{1}$ is unequal to $\mathrm{BB}_{1}$ :

$A A_{1}=R_{2} * \alpha$
$B B_{1}=R_{3} * \beta$

Besides, from the geometric relationships, we gets equations (3) and (4):

$\alpha=\theta+\beta$

$R_{2} * \beta+R_{1} * \theta=R_{2} * \alpha$

Transform Eq. (3) and Eq. (4), relationship of Eq. (5) is utilized.

$$
\alpha=\frac{R_{3}-R_{1}}{R_{2}-R_{1}} * \beta
$$

\subsection{Vibration equation}

As the disturbance from the ground to the lower plate is very slight, we assume the lower plate is static and the ball roll relative to the lower and upper plates. Be aware of that the mechanism will rotate when used lonely, so it is better to use in parallel with three or above.

When the mechanism is in motion state, the horizontal and vertical displaces $\mathrm{x}$ and $\mathrm{y}$ of the upper plate are

$$
\begin{aligned}
& \mathrm{x}=\left(R_{3}-R_{1}\right) \sin \beta+\left(R_{2}-R_{1}\right) \sin \alpha \\
& \mathrm{y}=\left(R_{2}+R_{3}-2 R_{1}\right)-\left[\left(R_{2}-R_{1}\right) \cos \alpha+\left(R_{3}-R_{1}\right) \cos \beta\right.
\end{aligned}
$$

Taking Eq. (5) into (6) and (7), the horizontal and vertical speeds $\dot{x}$ and $\dot{y}$ are obtained

$$
\begin{aligned}
& \dot{x}=\left(R_{3}-R_{1}\right)\left(\cos \beta+\cos \frac{R_{3}-R_{1}}{R_{2}-R_{1}} \beta\right) \dot{\beta} \\
& \dot{y}=\left(R_{3}-R_{1}\right)\left(\sin \beta+\sin \frac{R_{3}-R_{1}}{R_{2}-R_{1}} \beta\right) \dot{\beta}
\end{aligned}
$$

The synthetic speed $\mathrm{v}$ can be expressed as follow 


$$
\mathrm{v}=\sqrt{\dot{x}^{2}+\dot{y}^{2}}=\left(R_{3}-R_{1}\right) \dot{\beta} \sqrt{2+2 \cos \frac{R_{2}-R_{3}}{R_{2}-R_{1}} \beta}
$$

The kinetic energy of the upper plate is

$$
\mathrm{T}=\frac{1}{2} M v^{2}
$$

Where $\mathrm{M}$ is the quality of the upper plate. Assuming the potential energy of the upper plate is zero in balance state, then now we get potential energy at present

$$
\mathrm{V}=\text { Mgy }
$$

Equations (13) to (18) are achieved by Lagrange equation

$$
\begin{aligned}
& \mathrm{L}=\mathrm{T}-\mathrm{V} \\
& \frac{\partial L}{\partial \dot{\beta}}=M\left(R_{3}-R_{1}\right)^{2}\left(2+2 \cos \frac{R_{2}-R_{3}}{R_{2}-R_{1}} \beta\right) \dot{\beta} \\
& \frac{d}{d t}\left(\frac{\partial L}{\partial \dot{\beta}}\right)=M\left(R_{3}-R_{1}\right)^{2}\left(2+2 \cos \frac{R_{2}-R_{3}}{R_{2}-R_{1}} \beta\right) \ddot{\beta}+M\left(R_{3}-R_{1}\right)^{2}\left(-2 \frac{R_{2}-R_{3}}{R_{2}-R_{1}} \sin \frac{R_{2}-R_{3}}{R_{2}-R_{1}} \beta\right) \dot{\beta}^{2}
\end{aligned}
$$

Besides

$$
\frac{\partial L}{\partial \beta}=-M g\left(R_{3}-R_{1}\right)\left(\sin \beta+\sin \frac{R_{3}-R_{1}}{R_{2}-R_{1}} \beta\right)+M\left(R_{3}-R_{1}\right)^{2}\left(-\frac{R_{2}-R_{3}}{R_{2}-R_{1}} \sin \frac{R_{2}-R_{3}}{R_{2}-R_{1}} \beta\right) \dot{\beta}^{2}
$$

From Lagrange's equations

$M\left(R_{3}-R_{1}\right)^{2}\left(2+2 \cos \frac{R_{2}-R_{3}}{R_{2}-R_{1}} \beta\right) \ddot{\beta}+M g\left(R_{3}-R_{1}\right)\left(\sin \beta+\sin \frac{R_{3}-R_{1}}{R_{2}-R_{1}} \beta\right)-M\left(R_{3}-R_{1}\right)^{2}\left(-\frac{R_{2}-R_{3}}{R_{2}-R_{1}} \sin \frac{R_{2}-R_{3}}{R_{2}-R_{1}} \beta\right) \dot{\beta}^{2}=0$ theory

The third part of Eq. (17) is a higher order term and it can be omitted. From basic mathematical

$$
\left(\sin \beta+\sin \frac{R_{3}-R_{1}}{R_{2}-R_{1}} \beta\right)=2 \sin \frac{R_{2}+R_{3}-2 R_{1}}{2\left(R_{2}-R_{1}\right)} \beta \cos \frac{R_{2}-R_{3}}{2\left(R_{2}-R_{1}\right)} \beta
$$

As $\beta$ is very small, $\cos \frac{R_{2}-R_{3}}{2\left(R_{2}-R_{1}\right)} \beta \approx 1$, Eq. (18) turns into Eq. (19)

$$
\sin \beta+\sin \frac{R_{3}-R_{1}}{R_{2}-R_{1}} \beta=2 \sin \frac{R_{2}+R_{3}-2 R_{1}}{2\left(R_{2}-R_{1}\right)} \beta
$$

Taking Eq. (19) into (17)

$$
M\left(R_{3}-R_{1}\right)^{2}\left(2+2 \cos \frac{R_{2}-R_{3}}{R_{2}-R_{1}} \beta\right) \ddot{\beta}+2 \mathrm{Mg}\left(R_{3}-R_{1}\right) \sin \frac{R_{2}+R_{3}-2 R_{1}}{2\left(R_{2}-R_{1}\right)} \beta=0
$$

As above, $\cos \frac{R_{2}-R_{3}}{R_{2}-R_{1}} \beta=1, \sin \frac{R_{2}+R_{3}-2 R_{1}}{2\left(R_{2}-R_{1}\right)} \beta \approx \frac{R_{2}+R_{3}-2 R_{1}}{2\left(R_{2}-R_{1}\right)} \beta$, Eq. (20) is simplified as follow

$$
4\left(R_{3}-R_{1}\right) \ddot{\beta}+g \frac{R_{2}+R_{3}-2 R_{1}}{R_{2}-R_{1}} \beta=0
$$

The horizontal natural frequency is obtained

$$
\mathrm{f}=\frac{1}{4 \pi} \sqrt{\frac{g\left(R_{2}+R_{3}-2 R_{1}\right)}{\left(R_{2}-R_{1}\right)\left(R_{3}-R_{1}\right)}}
$$

\section{Simulations and experiments}

\subsection{Simulation}

Set the range of $R_{1}$ is from 0 to $0.05 \mathrm{~m}$, and the radii of the upper and the lower ball sockets changes in the range of $R_{1}$ to $0.5 \mathrm{~m}$. The Influence of $R_{1}$ and $R_{2}\left(R_{2}=R_{3}\right)$ on natural frequency is shown in Fig. 2, and the Influence of $R_{2}$ and $R_{3}$ on natural frequency when $R_{1}$ is $0.005 \mathrm{~m}, 0.01 \mathrm{~m}$ and $0.02 \mathrm{~m}$, respectively, are shown in Fig. 3 to fig.5. 


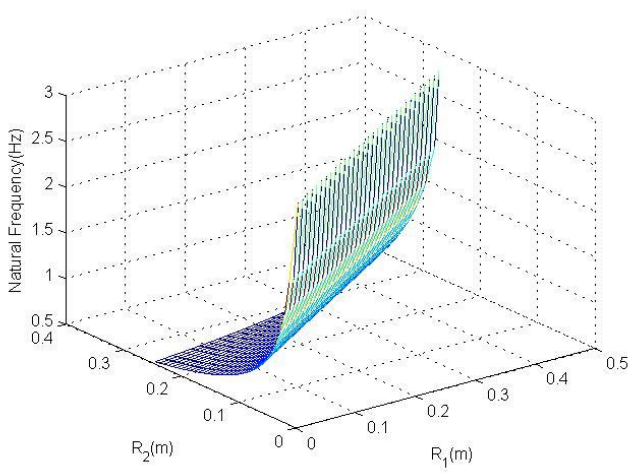

Fig. 2 Influence of $\mathrm{R}_{1}$ and $\mathrm{R}_{2}\left(\mathrm{R}_{2}=\mathrm{R}_{3}\right)$ on natural frequency

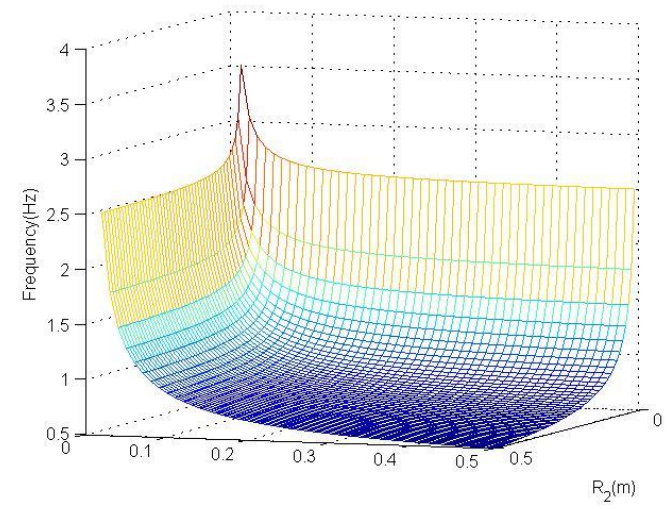

Fig.4 Influence of $\mathrm{R}_{2}$ and $\mathrm{R}_{3}$ on natural frequency under the condition: $\mathrm{R}_{1}=0.01 \mathrm{~m}$

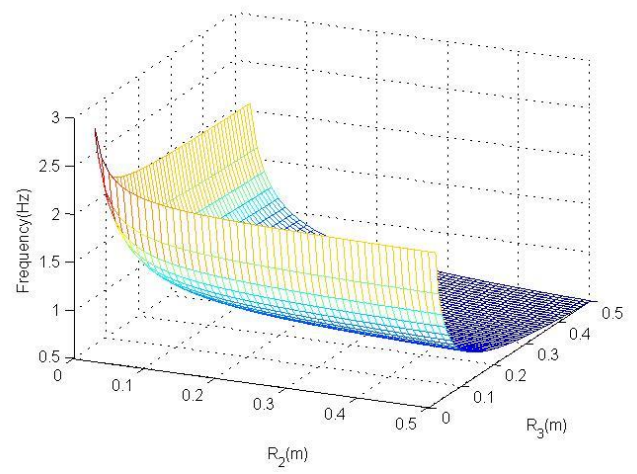

Fig. 3 Influence of $R_{2}$ and $R_{3}$ on natural frequency under the condition: $R_{1}=0.005 \mathrm{~m}$

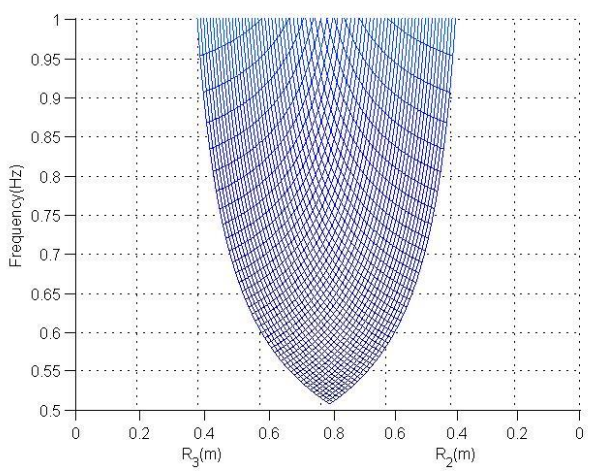

Fig.5 Influence of $\mathrm{R}_{2}$ and $\mathrm{R}_{3}$ on natural frequency under the condition: $\mathrm{R}_{1}=0.02 \mathrm{~m}$

Through the simulation analysis, it can be seen that the vibration natural frequency of the mechanism $f$ decreases with $R_{2}$ and $R_{3}$ increase, but the speed decreases; $f$ decreases with $R_{1}$ decreases, and the speed decreases. In addition, within the scope the lowest natural frequency can be close to $0.5 \mathrm{~Hz}$ and with the increase of $\mathrm{R}_{1}$, the lowest natural frequency increases slightly, but the variation is very small. If the natural frequency achieves sub- $\mathrm{Hz}$ only when $\mathrm{R}_{2}$ and $\mathrm{R}_{3}$ are minimum close to $0.4 \mathrm{~m}$.

\subsection{Experiments design}

For stability considerations, the design is shown in Fig.6. According to the actual situation, the material of the ball is 45 steel and $R_{1}$ is $0.02 \mathrm{~m}$, meanwhile the material of the upper and lower ball sockets is aluminum alloy $6061 \mathrm{~T}$ and $\mathrm{R}_{3}$ and $\mathrm{R}_{2}$ are $0.3 \mathrm{~m}$ and $0.4 \mathrm{~m}$. The upper and lower sheet metal plates of the vibration isolation system are aluminum alloy 6061T, between the two plates are four parallel mechanisms with symmetrical distribution in a rectangular form, and the lower sheet metal places on the ground, and the upper sheet metal supports the load. The experiment use BK (Bruel\& Kjær) as the vibration measurement system, and the vibration of the ground is the input signal and the vibration of the upper plate is the output signal, then the experimental setup is shown in Fig. 7. The experiments were divided into four groups and the corresponding load are $10 \mathrm{Kg}, 20 \mathrm{Kg}, 40 \mathrm{Kg}$ and $80 \mathrm{Kg}$, respectively. Each group test 3 times and then make date fitting by Matlab after the experimental results are averaged. The $\mathrm{x}$-axis represents the ground vibration frequency and the $\mathrm{y}$ axis represents the acceleration transfer ratio. 


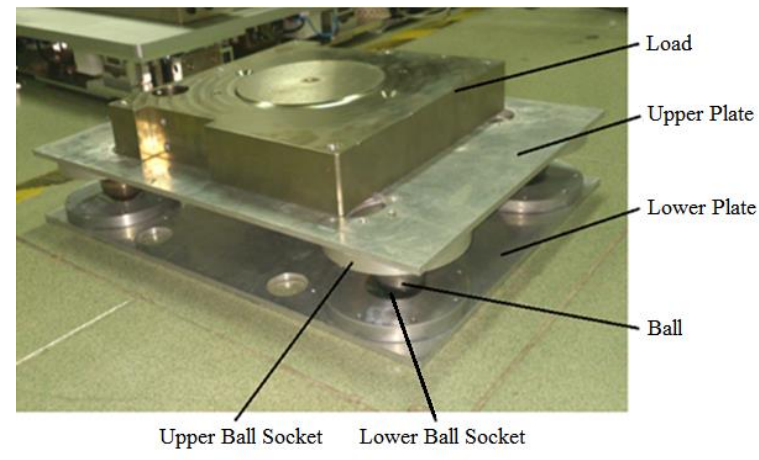

Fig.6 Photograph of the rolling ball-ball vibration isolation system

\subsection{Experimental consequences}

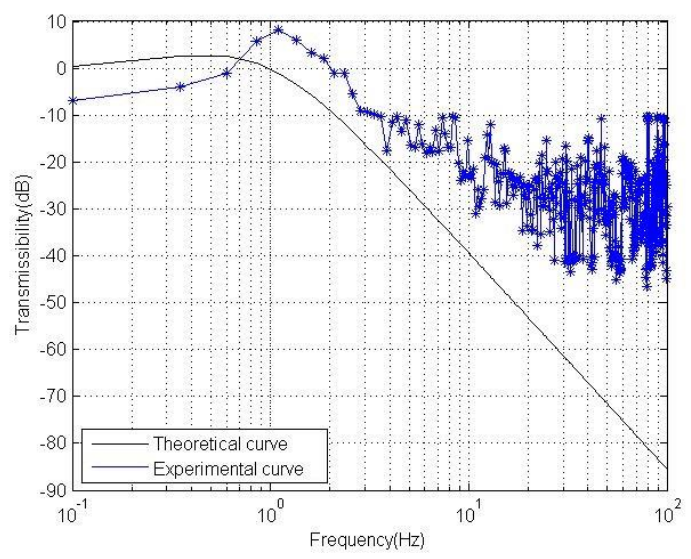

Fig.8 The theoretical and measured consequences when the payload $\mathrm{M}$ is $10 \mathrm{Kg}$

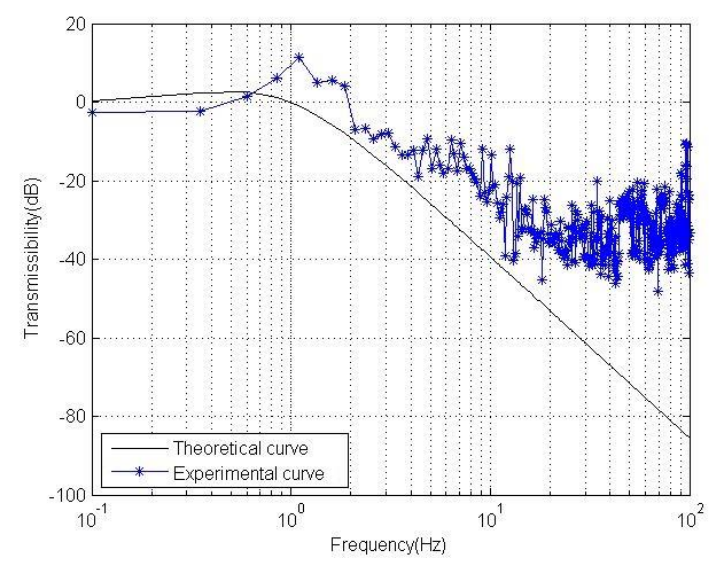

Fig.10 The theoretical and measured consequences when the payload $\mathrm{M}$ is $40 \mathrm{Kg}$

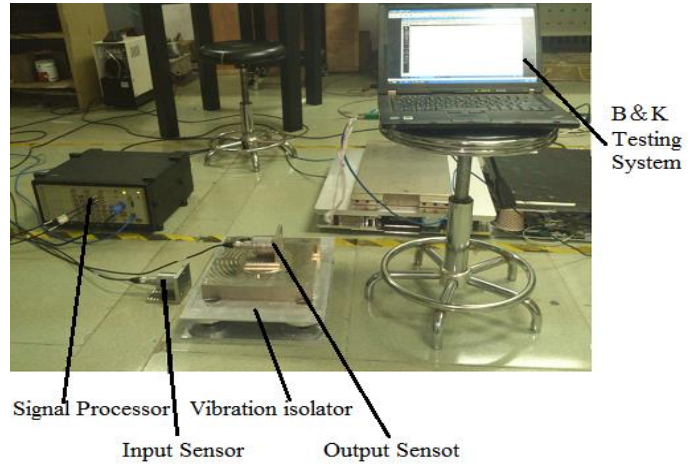

Fig.7 Photograph of the experimental system

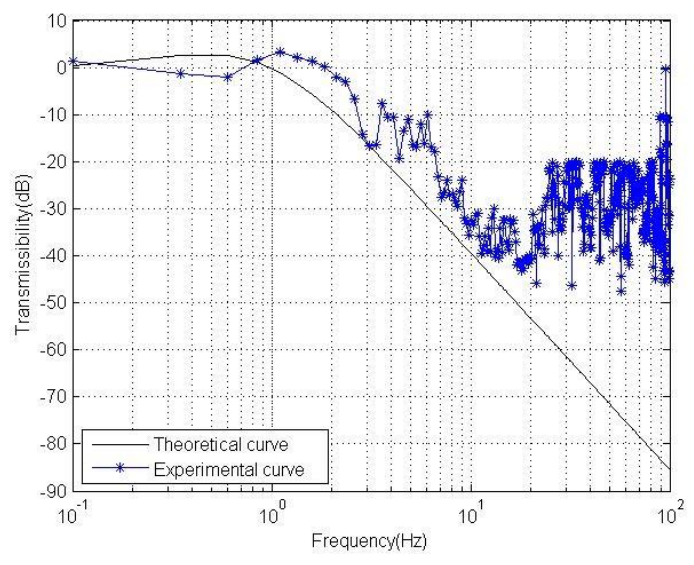

Fig.9 The theoretical and measured consequences when the payload $\mathrm{M}$ is $20 \mathrm{Kg}$

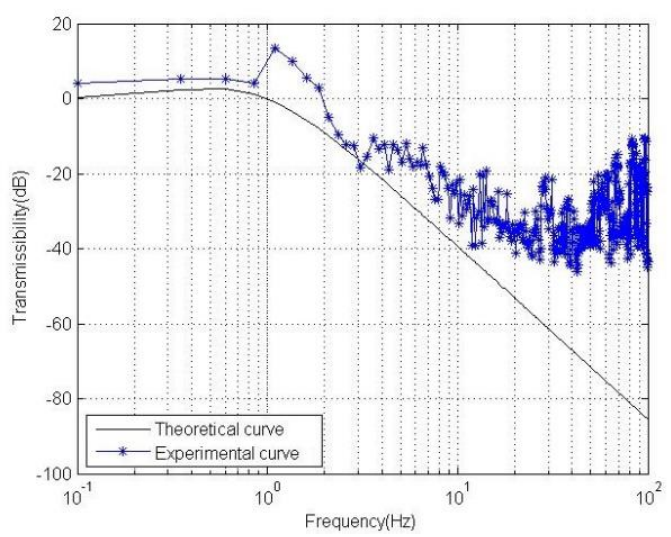

Fig.11 The theoretical and measured consequences when the payload $\mathrm{M}$ is $80 \mathrm{Kg}$

It can be seen from Fig. 8 to Fig. 11 that the natural frequency of experimental results are about to $1 \mathrm{~Hz}$, which are close to theoretical results $0.62 \mathrm{~Hz}$, and it may be due to the deviation between the hypothesis and the practice. Besides, it also can be seen the natural frequency of the system will not change with variable payload. The vibration transmissibility reaches to $-20 \mathrm{~dB}$ before the excitation frequency reaches to $10 \mathrm{~Hz}$, which confirms that the vibration isolation system is efficient.

\section{Conclusion}

This paper studied a rolling ball mechanism that the ball rolls relative to two ball sockets with unequal radii theoretically. Then we explore further the performance of vibration isolation system 
which composed of a plurality of the mechanisms. Experiments showed that the vibration transmissibility has reached to $-20 \mathrm{~dB}$ before the excitation frequency of the ground reaches to $10 \mathrm{~Hz}$, and the author is trying to employ active control technology to optimize further the performance of vibration isolation. In addition, the damping effect caused by the contact problem is also discussing. The vibration isolation system can be applied to the field of ultra-precision instrument, high-end physical and chemical experiments, which has a guiding significance for theory analysis and engineering design of the rolling ball vibration isolation mechanism.

\section{Acknowledgment}

This work was supported by National Natural Science Foundation of China-Study on Multi-degree Crosstalk Analysis and Finer Decoupling Control of Nanometer Accuracy Motion (No.51475261) and Beijing City Board of Education Foundation-Technology Development of High-performance Active Desktop Vibration Isolator (No.41)

\section{References}

[1] D.B.Debra. Vibration isolation of precision machine tools and instruments. CIRP Annals, 1992, 41(2), 711-718.

[2] Zhang Liguo, Zhang Jiazhong, Jia Liping, et al. Future and development of air springs [J].Journal of Vibration and Shock,2007,26(2):146-154.

[3] Erin C and Wilson B. An improved model of a pneumatic vibration isolator theory and experiment [J].Journal of Sound and Vibration, 1998, 218 (1):81-101.

[4] Lin, T. and W. and Hone, C. C. (1993). Base isolation by free rolling rods under basement [J].Earthquake Engineering and Structural Dynamics, Vol. 22, No. 3, pp. 261-273.

[5] Qiang Zhou. Experimental study and numerical analysis on structure base-isolated by ball system with restoring property [D].Thesis of the Master's Degree. Xi'an University of Architecture \& Technology, Xi'an, China, 1997.

[6] R.S.Jangid and Y.B.Londhe. Effectiveness of elliptical rolling rods for base isolation [J].J.Struct.Eng, 1998, 124:469-472.

[7] R.S.Jangid. Stochastic seismic response of structures isolated by rolling rods [J].Engineering Structures, 2000, 22:937-946.

[8] Masashi. Yasuda. Vibration control unit and vibration control body [P].US 20030052247A1, 2003.

[9] J.W.Butterworth. Seismic response of a non-concentric rolling isolator system [J].Advances in Structural Engineering, 2006, 9(1).

[10] P.S.Harvey Jr., R. Wiebe,H.P.Gavin. On the chaotic response of a nonlinear rolling isolation system [J]. Physica D, 256-257 (2013): 36-42.

[11] P.Scott Harvey Jr. and Henri P.Gavin. The nonholonomic and chaotic nature of a rolling isolation system [J].Journal of Sound and Vibration, 332 (2013):3535-3551. 